\title{
About Resolvability of the Problem of Lightning Coordinates Finding by the Time of Lightning Discharge Arrival
}

\author{
Yuriy Reingoldovich Shpadi ${ }^{1,2 *}$, Alexandr Sergeevich Inchin ${ }^{1}$, Anatoliy Yurievich Lozbin 1 , \\ Galymzhan Ayazbayev', Maxim Yurievich Shpadi', Ludmila Ismailovna Mailibayeva1
}

${ }^{1}$ Institute of Space Techniques and Technologies, Kazakhstan, Almaty

${ }^{2}$ Institute of Mathematics and Mathematical Modeling, Kazakhstan, Almaty

Email: *yu-shpadi@yandex.ru

How to cite this paper: Shpadi, Y. R., Inchin, A. S., Lozbin, A. Y., Ayazbayev, G., Shpadi, M. Y., \& Mailibayeva, L. I. (2021). About Resolvability of the Problem of Lightning Coordinates Finding by the Time of Lightning Discharge Arrival. Journal of Geoscience and Environment Protection, 9, 209-221. https://doi.org/10.4236/gep.2021.93013

Received: February 24, 2021

Accepted: March 27, 2021

Published: March 30, 2021

Copyright $\odot 2021$ by author(s) and Scientific Research Publishing Inc. This work is licensed under the Creative Commons Attribution International License (CC BY 4.0).

http://creativecommons.org/licenses/by/4.0/

\section{(c) (i) Open Access}

\begin{abstract}
On the unit sphere, the geometric problem of calculating the position of a point relative to three given points is considered. We know the length of three spherical segments that go out from the given points in the direction of the unknown point. The requirement must be fulfilled: the distance from each point to an unknown point must be equal to the sum of the length of the segment outgoing from this point, and some increment, the same for all three segments. In the article, the conditions for the solvability of a geometric problem are established by the methods of spherical trigonometry and vector algebra. It is proved that when they are fulfilled, the problem is always solvable. The number of solutions is two, except in rare cases where there is only one solution. A solution method is presented. One of the practical applications is the problem of determining the time and location of a cloud-to-ground lightning discharge, which is directly reduced to this problem.
\end{abstract}

\section{Keywords}

Meteorology, Lightning Discharge Positioning, Spherical Trigonometry, Vector Algebra, Algorithms

\section{Introduction}

The article (Lozbin, Shpadi, \& Inchin, 2016) proposes an algorithm for calculating the location and time of a lightning discharge using the Time-of-Arrival (ToA) method for three stations. However, this algorithm is not sufficiently mathematically substantiated. This shortcoming is addressed in this article. In 
addition, this article proposes a new algorithm for solving the problem, which is simpler for numerical calculations than the previous one (Lozbin, Shpadi, \& Inchin, 2016).

The mathematical model of the problem under consideration is implemented on the unit sphere. In this regard, we note some provisions of the geometry on the sphere, which we will use (Krantz, 2007; Volynsky, 1977).

A sphere, its radius is one which is called a unit sphere.

A great circle of a sphere is a circle cut by a sphere from a plane passing through its center.

A spherical segment is an arc of a circle of a great circle. The length of the spherical segment of the unit sphere is measured by a central angle, the sides of which pass through the ends of the spherical segment.

The distance between two points of a sphere is the length of a smaller spherical segment connecting these points.

A spherical triangle is a part of a sphere bounded by three spherical segments with common vertices.

A spherical triangle, the length of each side of which is strictly less than the length of the semicircle of the great circle, is called Euler's triangle. For an Euler triangle, the following statement is true: the sum of the lengths of its two sides is greater than the length of the third side.

A spherical triangle whose area is zero is called a degenerate spherical triangle. All of its vertices are located on the same circumference of a great circle and can coincide. In this article, a degenerate spherical triangle appears as a special case in problems with parameters that determine the values of its sides and angles.

\section{Formulation of the Problem}

Consider a unit sphere with a center coinciding with the origin of the Cartesian system $O x y z$. We introduce a coordinate system $(\varphi, \theta)$ on the sphere, where $\varphi$ and $\theta$ are longitude and latitude of the points of the sphere respectively, $-\pi<\varphi \leq \pi, 0 \leq \theta \leq \pi$. In accordance with the concepts of a geographic coordinate system, points with latitude $\theta=0$ and $\theta=\pi$ we will be called the North and South poles, and the arcs connecting them as meridians. In addition, let the equator be points of a circle on a sphere with latitude $\theta=\pi / 2$.

Consider Euler's triangle, the vertices of which are at the points $P_{i}\left(\varphi_{i}, \theta_{i}\right)$, $i=1,2,3$. Let $P_{s}\left(\varphi_{s}, \theta_{s}\right)$ be an unknown point on the sphere, the position of which is to be found. Let $\alpha_{i}, i=1,2,3$, be spherical segments of a given length going out from points $P_{i}\left(\varphi_{i}, \theta_{i}\right)$ in the direction of an unknown point $P_{s}\left(\varphi_{s}, \theta_{s}\right)$. The length of each segment is also denoted by $\alpha_{i}$. Such a combination of concepts will do not cause a semantic contradiction.

We denote as $\Delta \alpha$ the increment of the lengths for all segments $\alpha_{i}$. This increment $\Delta \alpha$, which is the same for all segments $\alpha_{i}$, can be a positive or negative number, as well as zero.

The considered problem is a determination on the sphere the point $P_{s}\left(\varphi_{s}, \theta_{s}\right)$ and the value of increment $\Delta \alpha$ of the segments $\alpha_{i}$ in accordance with the 
requirement: the spherical distance from the vertices $P_{i}\left(\varphi_{i}, \theta_{i}\right)$ to the point $P_{s}\left(\varphi_{s}, \theta_{s}\right)$ must be equal to the sum $\alpha_{i}+\Delta \alpha, i=1,2,3$.

\section{Task Analysis}

To solve the problem, we will use vector algebra (Beklemishev, 2008). The one-to-one correspondence between Cartesian and spherical coordinates of points of a unit sphere is determined by the following equations:

$$
\left\{\begin{array}{l}
x=\cos \varphi \sin \theta \\
y=\sin \varphi \sin \theta \\
z=\cos \theta
\end{array}\right.
$$

We introduce unit radius vectors $\boldsymbol{r}_{i}=\left\{x_{i}, y_{i}, z_{i}\right\}$ of the vertices $P_{i}\left(\varphi_{i}, \theta_{i}\right)$ of $P_{1} P_{2} P_{3}$ triangle and unit radius vector $\boldsymbol{r}_{s}=\left\{x_{s}, y_{s}, z_{s}\right\}$ of the target $P_{s}\left(\varphi_{s}, \theta_{s}\right)$ point. The criterion for the non-degeneracy of the $P_{1} P_{2} P_{3}$ triangle is the non-zero value of the triple product of three vectors $r_{i}=\left\{x_{i}, y_{i}, z_{i}\right\}$. Therefore the condition

$$
\left(\boldsymbol{r}_{1}, \boldsymbol{r}_{2}, \boldsymbol{r}_{3}\right)=\boldsymbol{r}_{1} \cdot\left(\boldsymbol{r}_{2} \times \boldsymbol{r}_{3}\right) \neq 0
$$

must be met, where the symbol ". " and " $x$ " are scalar and vector products of vectors.

Since the $P_{1} P_{2} P_{3}$ triangle is by definition Eulerian, the following inequalities hold:

$$
0<\arccos \left(\boldsymbol{r}_{i} \cdot \boldsymbol{r}_{j}\right)<\pi, \quad i, j=1,2,3, \quad i \neq j,
$$

where $\arccos \left(\boldsymbol{r}_{i} \cdot \boldsymbol{r}_{j}\right)$ determines the angle between unit vectors $\boldsymbol{r}_{i}$ and $\boldsymbol{r}_{j}$, and distance between points $P_{i}$ and $P_{j}$ equal to this angle.

Thus, the task is reduced to solving the system of equations:

$$
\left\{\begin{array}{l}
\arccos \left(\boldsymbol{r}_{1} \cdot \boldsymbol{r}_{s}\right)=\alpha_{1}+\Delta \alpha, \\
\arccos \left(\boldsymbol{r}_{2} \cdot \boldsymbol{r}_{s}\right)=\alpha_{2}+\Delta \alpha, \\
\arccos \left(\boldsymbol{r}_{3} \cdot \boldsymbol{r}_{s}\right)=\alpha_{3}+\Delta \alpha,
\end{array}\right.
$$

with three unknown $\varphi_{s}, \theta_{s}$ and $\Delta \alpha$.

Next, we assume that the numbering of the vertices $P_{i}$ of the $P_{1} P_{2} P_{3}$ triangle is selected taking into account inequalities:

$$
\alpha_{1} \leq \alpha_{2} \leq \alpha_{3} .
$$

Theorem 1. In order for the system of Equations (4) to have some solution in the conditions of the above problem, it is necessary to fulfill the next conditions:

$$
\arccos \left(\boldsymbol{r}_{i} \cdot \boldsymbol{r}_{j}\right) \geq \alpha_{j}-\alpha_{i}, \quad i, j=1,2,3, \quad i<j .
$$

Proof. First, we note that condition (5) implies inequalities

$$
\alpha_{2}-\alpha_{1} \geq 0, \quad \alpha_{3}-\alpha_{1} \geq 0, \quad \alpha_{3}-\alpha_{2} \geq 0 .
$$

Suppose that as a result of the solution of the system (4), the position of the point $P_{s}$ is determined. In this case, for each possibly degenerate, triangle $P_{s} P_{i} P_{j}$ the inequalities must be satisfied: 


$$
\arccos \left(\boldsymbol{r}_{i} \cdot \boldsymbol{r}_{j}\right) \geq \arccos \left(\boldsymbol{r}_{j} \cdot \boldsymbol{r}_{s}\right)-\arccos \left(\boldsymbol{r}_{i} \cdot \boldsymbol{r}_{s}\right), \quad i, j=1,2,3, \quad i<j .
$$

Substituting in the right part of inequalities (8) the values of arc cosines from (4), we get a system of inequalities (6). "

Theorem 2. For solvability of the system of Equations (4), the lengths of the $\alpha_{1}, \alpha_{2}, \alpha_{3}$ segments must satisfy the conditions

$$
0 \leq \alpha_{j}-\alpha_{i}<\pi, \quad i, j=1,2,3, \quad i<j .
$$

Proof. The non-negativity of the difference $\alpha_{j}-\alpha_{i}$ in (9) follows from inequalities (5). In case of $\alpha_{j}-\alpha_{i} \geq \pi$, the corresponding inequality in (6) will contradict conditions (3).

\section{Solution Algorithm}

We consider the algorithm of solution of system (4), assuming that the segments $\alpha_{1}, \alpha_{2}, \alpha_{3}$ are satisfied the inequalities (5) and (9), and the inequalities (6) are satisfied in a strict sense, that is:

$$
\arccos \left(\boldsymbol{r}_{i} \cdot \boldsymbol{r}_{j}\right)>\alpha_{j}-\alpha_{i}, \quad i, j=1,2,3, \quad i<j .
$$

Without losing generality, we will assume that the point $P_{1}\left(\varphi_{1}, \theta_{1}\right)$ is at the North pole, that is $\theta_{1}=0$. In this case, the radius vector of the point $P_{1}\left(\varphi_{1}, \theta_{1}\right)$ is

$$
\boldsymbol{r}_{1}=\{0,0,1\} .
$$

If the point $P_{1}\left(\varphi_{1}, \theta_{1}\right)$ does not coincide with the North pole, then we should move the triangle $P_{1} P_{2} P_{3}$ around the sphere by orthogonal transformation:

$$
\boldsymbol{Q}=\boldsymbol{Q}_{\theta} \boldsymbol{Q}_{\varphi},
$$

which must be applied to radius vectors $\boldsymbol{r}_{1}, \boldsymbol{r}_{2}, \boldsymbol{r}_{3}$. The matrices $\boldsymbol{Q}_{\varphi}$ and $\boldsymbol{Q}_{\theta}$ are defined by the longitude and latitude of the point $P_{1}$ and are written as:

$$
\boldsymbol{Q}_{\varphi}=\left(\begin{array}{ccc}
\cos \varphi_{1} & \sin \varphi_{1} & 0 \\
-\sin \varphi_{1} & \cos \varphi_{1} & 0 \\
0 & 0 & 1
\end{array}\right), \boldsymbol{Q}_{\theta}=\left(\begin{array}{ccc}
\cos \theta_{1} & 0 & -\sin \theta_{1} \\
0 & 1 & 0 \\
\sin \theta_{1} & 0 & \cos \theta_{1}
\end{array}\right)
$$

The matrix $\boldsymbol{Q}_{\varphi}$ rotates the triangle points about the axis $\mathrm{Oz}$ by an angle $-\varphi_{1}$, and the matrix $\boldsymbol{Q}_{\theta}$ rotates about the axis $O y$ by an angle $-\theta_{1}$. As the result of these rotations, the triangle $P_{1} P_{2} P_{3}$ moves along the surface of the sphere, with preserving the lengths of the sides and the values of the angles, as well as the direction of the border so that the point $P_{1}$ is at the North pole.

After solving the system (4) and finding the point $P_{s}$, the inverse transformation

$$
\boldsymbol{Q}^{\mathrm{T}}=\boldsymbol{Q}_{\varphi}^{\mathrm{T}} \boldsymbol{Q}_{\theta}^{\mathrm{T}},
$$

applied to the radius vector $\boldsymbol{r}_{s}=\left\{x_{s}, y_{s}, z_{s}\right\}$ will restore the location of the point $P_{s}$ relative to the original position of the spherical triangle $P_{1} P_{2} P_{3}$. The symbol "T" in (14) means the transposition of matrices.

Subtracting the first equation of system (4) from the second and third, we ob- 
tain a system of two equations

$$
\left\{\begin{array}{l}
\arccos \left(\boldsymbol{r}_{2} \cdot \boldsymbol{r}_{s}\right)-\arccos \left(\boldsymbol{r}_{1} \cdot \boldsymbol{r}_{s}\right)=\alpha_{2}-\alpha_{1} \\
\arccos \left(\boldsymbol{r}_{3} \cdot \boldsymbol{r}_{s}\right)-\arccos \left(\boldsymbol{r}_{1} \cdot \boldsymbol{r}_{s}\right)=\alpha_{3}-\alpha_{1}
\end{array}\right.
$$

in which only two spherical coordinates $\varphi_{s}$ and $\theta_{s}$ of the points $P_{s}$ are unknown.

Taking to account (11), we obtain:

$$
\boldsymbol{r}_{1} \cdot \boldsymbol{r}_{\mathrm{s}}=z_{\mathrm{s}}=\cos \theta_{\mathrm{s}}, \quad 0 \leq \theta_{\mathrm{s}} \leq \pi .
$$

The system (15) taking into account (16) be as

$$
\left\{\begin{array}{l}
\arccos \left(\boldsymbol{r}_{2} \cdot \boldsymbol{r}_{s}\right)=\theta_{s}+\alpha_{2}-\alpha_{1} \\
\arccos \left(\boldsymbol{r}_{3} \cdot \boldsymbol{r}_{s}\right)=\theta_{s}+\alpha_{3}-\alpha_{1}
\end{array}\right.
$$

Calculating the cosines of the left and right parts of the equations in (17), we obtain:

$$
\left\{\begin{array}{l}
\boldsymbol{r}_{2} \cdot \boldsymbol{r}_{s}=\cos \left(\theta_{s}+\alpha_{2}-\alpha_{1}\right) \\
\boldsymbol{r}_{3} \cdot \boldsymbol{r}_{s}=\cos \left(\theta_{s}+\alpha_{3}-\alpha_{1}\right)
\end{array}\right.
$$

Let us expand the scalar products of vectors in (18)

$$
\boldsymbol{r}_{i} \cdot \boldsymbol{r}_{s}=x_{i} x_{s}+y_{i} y_{s}+z_{i} z_{s}=x_{i} \cos \varphi_{s} \sin \theta_{s}+y_{i} \sin \varphi_{s} \sin \theta_{s}+z_{i} \cos \theta_{s},
$$

and let us convert the right parts in (18) by formula of cosine of two angles sum

$$
\cos \left(\theta_{s}+\alpha_{i}-\alpha_{1}\right)=\cos \theta_{s} \cos \left(\alpha_{i}-\alpha_{1}\right)-\sin \theta_{s} \sin \left(\alpha_{i}-\alpha_{1}\right) \text {. }
$$

We write the equations of system (18) in the form of equalities to zero of two linear combinations of functions $\sin \theta_{s}$ and $\cos \theta_{s}$

$$
\left\{\begin{array}{l}
{\left[x_{2} \cos \varphi_{s}+y_{2} \sin \varphi_{s}+\sin \left(\alpha_{2}-\alpha_{1}\right)\right] \sin \theta_{s}+\left[z_{2}-\cos \left(\alpha_{2}-\alpha_{1}\right)\right] \cos \theta_{s}=0} \\
{\left[x_{3} \cos \varphi_{s}+y_{3} \sin \varphi_{s}+\sin \left(\alpha_{3}-\alpha_{1}\right)\right] \sin \theta_{s}+\left[z_{3}-\cos \left(\alpha_{3}-\alpha_{1}\right)\right] \cos \theta_{s}=0}
\end{array}\right.
$$

Since the functions $\sin \theta_{s}$ and $\cos \theta_{s}$ do not simultaneously vanish for any values of $\theta_{s}$ due to the identity $\sin ^{2} \theta_{s}+\cos ^{2} \theta_{s} \equiv 1$, then for the existence of a solution $\theta_{s}$ to system (19) its determinant must be equal to zero, i.e.,

$$
\left|\begin{array}{ll}
x_{2} \cos \varphi_{s}+y_{2} \sin \varphi_{s}+\sin \left(\alpha_{2}-\alpha_{1}\right) & z_{2}-\cos \left(\alpha_{2}-\alpha_{1}\right) \\
x_{3} \cos \varphi_{s}+y_{3} \sin \varphi_{s}+\sin \left(\alpha_{3}-\alpha_{1}\right) & z_{3}-\cos \left(\alpha_{3}-\alpha_{1}\right)
\end{array}\right|=0 .
$$

Calculating the determinant in (20) and selecting in it a linear combination of functions $\sin \varphi_{s}$ and $\cos \varphi_{s}$, we obtain the equation for $\varphi_{s}$ :

$$
\begin{aligned}
& \left\{x_{2}\left[z_{3}-\cos \left(\alpha_{3}-\alpha_{1}\right)\right]-x_{3}\left[z_{2}-\cos \left(\alpha_{2}-\alpha_{1}\right)\right]\right\} \cos \varphi_{s} \\
& +\left\{y_{2}\left[z_{3}-\cos \left(\alpha_{3}-\alpha_{1}\right)\right]-y_{3}\left[z_{2}-\cos \left(\alpha_{2}-\alpha_{1}\right)\right]\right\} \sin \varphi_{s} \\
& =z_{2} \sin \left(\alpha_{3}-\alpha_{1}\right)-z_{3} \sin \left(\alpha_{2}-\alpha_{1}\right)-\sin \left(\alpha_{3}-\alpha_{2}\right)
\end{aligned}
$$

Let us introduce the following notation

$$
\begin{aligned}
& A_{\varphi}=x_{2}\left[z_{3}-\cos \left(\alpha_{3}-\alpha_{1}\right)\right]-x_{3}\left[z_{2}-\cos \left(\alpha_{2}-\alpha_{1}\right)\right], \\
& B_{\varphi}=y_{2}\left[z_{3}-\cos \left(\alpha_{3}-\alpha_{1}\right)\right]-y_{3}\left[z_{2}-\cos \left(\alpha_{2}-\alpha_{1}\right)\right],
\end{aligned}
$$




$$
C_{\varphi}=z_{2} \sin \left(\alpha_{3}-\alpha_{1}\right)-z_{3} \sin \left(\alpha_{2}-\alpha_{1}\right)-\sin \left(\alpha_{3}-\alpha_{2}\right),
$$

and write an Equation (21) as

$$
A_{\varphi} \cos \varphi_{s}+B_{\varphi} \sin \varphi_{s}=C_{\varphi} .
$$

We show, that

$$
A_{\varphi}^{2}+B_{\varphi}^{2}>0 .
$$

Suppose that the opposite is true, that is, that the equality

$$
A_{\varphi}=B_{\varphi}=0 .
$$

From the conditions (10) and equations

$$
\boldsymbol{r}_{i} \cdot \boldsymbol{r}_{1}=z_{i}, \quad i=2,3
$$

we get that

$$
z_{i}-\cos \left(\alpha_{i}-\alpha_{1}\right)<0, \quad i=2,3 .
$$

If we now consider Equations (27) taking into account (22)-(23) as a linear homogeneous system of equations in which expressions $z_{i}-\cos \left(\alpha_{i}-\alpha_{1}\right)$, $i=2,3$, are unknown, then in view of (29) its determinant must be zero, that is,

$$
x_{2} y_{3}-x_{3} y_{2}=0 \text {. }
$$

This equality means that the projection of the vector product $\boldsymbol{r}_{2} \times \boldsymbol{r}_{3}$ on the axis $\mathrm{Oz}$ is zero. It follows that the plane of the vectors $\boldsymbol{r}_{2}$ and $\boldsymbol{r}_{3}$ passes through both poles of the sphere. As a result, we get that all three vectors $\boldsymbol{r}_{1}, \boldsymbol{r}_{2}$ and $\boldsymbol{r}_{3}$ belong to the same plane, and this contradicts the condition (2) of non-degeneracy of the triangle $P_{1} P_{2} P_{3}$. Thus, assumption (27) is not true and inequality (26) under conditions (10) be valid for any triangle $P_{1} P_{2} P_{3}$.

By defining a value of parameter $\varsigma \in(-\pi, \pi]$ from a system of equations

$$
\left\{\begin{array}{l}
\cos \varsigma=\frac{A_{\varphi}}{\sqrt{A_{\varphi}^{2}+B_{\varphi}^{2}}} \\
\sin \varsigma=\frac{B_{\varphi}}{\sqrt{A_{\varphi}^{2}+B_{\varphi}^{2}}}
\end{array}\right.
$$

and assume, that

$$
\Delta_{\varphi}=\frac{C_{\varphi}}{\sqrt{A_{\varphi}^{2}+B_{\varphi}^{2}}} .
$$

reduce Equation (25) to form

$$
\cos \left(\varphi_{s}-\varsigma\right)=\Delta_{\varphi} .
$$

In case of $\left|\Delta_{\varphi}\right| \leq 1$, the general solution of the Equation (32) will be as

$$
\varphi_{s}=\varsigma \pm \arccos \Delta_{\varphi}+2 k \pi, \quad k=0, \pm 1, \pm 2, \cdots,
$$

from which we need to select values $\varphi_{s}$ which are in the range $-\pi<\varphi_{s} \leq \pi$. In case of $\left|\Delta_{\varphi}\right|<1$, there will be two such solutions and, in the case of $\left|\Delta_{\varphi}\right|=1$ only one solution. If $\left|\Delta_{\varphi}\right|>1$, then the problem has no solutions. 
After finding longitude $\varphi_{s}$, the corresponding latitude value $\theta_{s}$ of the target point $P_{s}\left(\varphi_{s}, \theta_{s}\right)$, belonging to the interval $0 \leq \theta_{s} \leq \pi$ is determined from any equation of the system (19), that is,

$$
\operatorname{ctg} \theta_{s}=-\frac{x_{i} \cos \varphi_{s}+y_{i} \sin \varphi_{s}+\sin \left(\alpha_{i}-\alpha_{1}\right)}{z_{i}-\cos \left(\alpha_{i}-\alpha_{1}\right)} \text {, where } i=2 \text { or } i=3 \text {. }
$$

From (29) it follows that $z_{i}-\cos \left(\alpha_{i}-\alpha_{1}\right) \neq 0, i=2,3$. In this case, Equation (34) with unknown $\theta_{s}$ has only one solution on the interval $0<\theta_{s}<\pi$.

For calculating $\theta_{s}$ directly, it is convenient to use equivalent formulas

$$
\theta_{s}=\frac{\pi}{2}+\operatorname{arctg} \frac{x_{i} \cos \varphi_{s}+y_{i} \sin \varphi_{s}+\sin \left(\alpha_{i}-\alpha_{1}\right)}{z_{i}-\cos \left(\alpha_{i}-\alpha_{1}\right)}, i=2,3,
$$

since in computing environments for computers the arc cotangent function is usually absent.

The value $\Delta \alpha$ is determined from any equation of the system (4), that is,

$$
\Delta \alpha=\arccos \left(\boldsymbol{r}_{i} \cdot \boldsymbol{r}_{s}\right)-\alpha_{i}, \quad i=1,2,3 .
$$

Theorem 3. The inequality

$$
A_{\varphi}^{2}+B_{\varphi}^{2}-C_{\varphi}^{2} \geq 0
$$

is always satisfied, when $A_{\varphi}, B_{\varphi}$ and $C_{\varphi}$ are defined by expressions (22)-(24) and conditions (5), (6) and (9) are met.

Proof. The inequality (37) is proved by directly calculating its left part using the expressions (22)-(24) and transforming them identical. We have

$$
\begin{aligned}
A_{\varphi}^{2}+B_{\varphi}^{2}= & \left(x_{2}^{2}+y_{2}^{2}\right)\left[z_{3}-\cos \left(\alpha_{3}-\alpha_{1}\right)\right]^{2} \\
& -2\left(x_{2} x_{3}+y_{2} y_{3}\right)\left[z_{3}-\cos \left(\alpha_{3}-\alpha_{1}\right)\right]\left[z_{2}-\cos \left(\alpha_{2}-\alpha_{1}\right)\right] \\
& +\left(x_{3}^{2}+y_{3}^{2}\right)\left[z_{2}-\cos \left(\alpha_{2}-\alpha_{1}\right)\right]^{2}
\end{aligned}
$$

Applying identities

$$
\begin{gathered}
x_{2}^{2}+y_{2}^{2}=\left(x_{2}^{2}+y_{2}^{2}+z_{2}^{2}\right)-z_{2}^{2}=1-z_{2}^{2}, \\
x_{2} x_{3}+y_{2} y_{3}=\left(x_{2} x_{3}+y_{2} y_{3}+z_{2} z_{3}\right)-z_{2} z_{3}=\boldsymbol{r}_{2} \cdot \boldsymbol{r}_{3}-z_{2} z_{3}, \\
x_{3}^{2}+y_{3}^{2}=\left(x_{3}^{2}+y_{3}^{2}+z_{3}^{2}\right)-z_{3}^{2}=1-z_{3}^{2}, \\
\boldsymbol{r}_{2} \cdot \boldsymbol{r}_{3}=\left[\boldsymbol{r}_{2} \cdot \boldsymbol{r}_{3}-\cos \left(\alpha_{3}-\alpha_{2}\right)\right]+\cos \left(\alpha_{3}-\alpha_{2}\right)
\end{gathered}
$$

and taking into account (38), we have

$$
\begin{aligned}
A_{\varphi}^{2}+B_{\varphi}^{2}= & -2\left(\boldsymbol{r}_{2} \cdot \boldsymbol{r}_{3}-\cos \left(\alpha_{3}-\alpha_{2}\right)\right)\left[\boldsymbol{r}_{1} \cdot \boldsymbol{r}_{3}-\cos \left(\alpha_{3}-\alpha_{1}\right)\right] \\
& \times\left[\boldsymbol{r}_{1} \cdot \boldsymbol{r}_{2}-\cos \left(\alpha_{2}-\alpha_{1}\right)\right]-\cos \left(\alpha_{3}-\alpha_{1}\right)\left[z_{3}-\cos \left(\alpha_{3}-\alpha_{1}\right)\right] \\
& \times\left[z_{2}-\cos \left(\alpha_{2}-\alpha_{1}\right)\right]+2\left[z_{2}^{2} z_{3}^{2}-z_{2}^{2} z_{3} \cos \left(\alpha_{3}-\alpha_{1}\right)\right. \\
& \left.-z_{2} z_{3}^{2} \cos \left(\alpha_{2}-\alpha_{1}\right)+z_{2} z_{3} \cos \left(\alpha_{2}-\alpha_{1}\right) \cos \left(\alpha_{3}-\alpha_{1}\right)\right] \\
& +\left(1-z_{2}^{2}\right)\left[z_{3}^{2}-2 z_{3} \cos \left(\alpha_{3}-\alpha_{1}\right)+\cos ^{2}\left(\alpha_{3}-\alpha_{1}\right)\right] \\
& +\left(1-z_{3}^{2}\right)\left[z_{2}^{2}-2 z_{2} \cos \left(\alpha_{2}-\alpha_{1}\right)+\cos ^{2}\left(\alpha_{2}-\alpha_{1}\right)\right]
\end{aligned}
$$


Then, after a series of identical transformations taking into account (24), we have the final result

$$
\begin{aligned}
A_{\varphi}^{2}+B_{\varphi}^{2}-C_{\varphi}^{2}= & -2\left[\boldsymbol{r}_{1} \cdot \boldsymbol{r}_{2}-\cos \left(\alpha_{2}-\alpha_{1}\right)\right]\left[\boldsymbol{r}_{1} \cdot \boldsymbol{r}_{3}-\cos \left(\alpha_{3}-\alpha_{1}\right)\right] \\
& \times\left[\boldsymbol{r}_{2} \cdot \boldsymbol{r}_{3}-\cos \left(\alpha_{3}-\alpha_{2}\right)\right]
\end{aligned}
$$

Inequalities (6) under conditions (9) are equivalent to inequalities

$$
\boldsymbol{r}_{i} \cdot \boldsymbol{r}_{j} \leq \cos \left(\alpha_{j}-\alpha_{i}\right), \quad i, j=1,2,3, \quad i<j,
$$

from which the inequality (37) is coming. -

Corollary. System of Equations (15) always has two solutions if, under the conditions of Theorem 3, inequalities (10) are satisfied in the strict sense. Indeed, in case of satisfaction (10), the right part of (39) is positive and, hence, there is a strict $A_{\varphi}^{2}+B_{\varphi}^{2}-C_{\varphi}^{2}>0$ inequality, from which, taking into account (31), the $\left|\Delta_{\varphi}\right|<1$ is follows.

\section{Exceptional Cases of Solving the Problem}

Those cases of solution of Equation (25) when the equalities

$$
A_{\varphi}^{2}+B_{\varphi}^{2}-C_{\varphi}^{2}=0 \text {, }
$$

were called exceptional. Equality (40) occurs if at least one of the equalities

$$
\boldsymbol{r}_{i} \cdot \boldsymbol{r}_{j}=\cos \left(\alpha_{j}-\alpha_{i}\right), \quad i, j=1,2,3, \quad i<j,
$$

is fulfilled.

Theorem 4. If, with some $i$ and $j, i<j$, the equality (41) is fulfilled, then the vector $\boldsymbol{r}_{s}$ lies in a plane of vectors $\boldsymbol{r}_{i}$ and $\boldsymbol{r}_{j}$.

Proof. First, we emphasize that due to the non-degeneracy of the $P_{1} P_{2} P_{3}$ triangle, the vectors $\boldsymbol{r}_{i}$ and $\boldsymbol{r}_{j}, i, j=1,2,3, i \neq j$, are not collinear. Therefore,

$$
\boldsymbol{r}_{i} \cdot \boldsymbol{r}_{j} \neq \pm 1 .
$$

In this case, each pair of vectors $\boldsymbol{r}_{i}$ and $\boldsymbol{r}_{j}$ uniquely defines a certain plane. Note also that, a strict inequality $\alpha_{j}>\alpha_{i}, i<j$, follows from (5), (41) and (42).

Calculating the cosines of the left and right sides in (41), we obtain $\arccos \left(\boldsymbol{r}_{i} \cdot \boldsymbol{r}_{j}\right)=\alpha_{j}-\alpha_{i}$. Subtracting the $i$-th equation of system (4) from the $j$-th, $i<j$, and replacing the difference $\alpha_{j}-\alpha_{i}$ with $\arccos \left(\boldsymbol{r}_{i} \cdot \boldsymbol{r}_{j}\right)$, we obtain the equality

$$
\arccos \left(\boldsymbol{r}_{j} \cdot \boldsymbol{r}_{s}\right)-\arccos \left(\boldsymbol{r}_{i} \cdot \boldsymbol{r}_{s}\right)=\arccos \left(\boldsymbol{r}_{i} \cdot \boldsymbol{r}_{j}\right) .
$$

Consider a trihedral angle with edges $\boldsymbol{r}_{i}, \boldsymbol{r}_{j}$ and $\boldsymbol{r}_{s}$. Based on the theorem from stereometry (Kiselev, 2004) on the properties of the sum of two plane angles of a trihedral angle, from equality (43) we obtain that the trihedral angle with edges $\boldsymbol{r}_{i}, \boldsymbol{r}_{j}$ and $\boldsymbol{r}_{\mathrm{s}}$ is degenerate and, therefore, the vector $\boldsymbol{r}_{\mathrm{s}}$ lies in the plane of the vectors $\boldsymbol{r}_{i}$ and $\boldsymbol{r}_{j}$.

Consider the proof of Theorem 4 based on vector algebra and find a formula 
for calculating the vector $\boldsymbol{r}_{s}$. We introduce the notations $\alpha=\arccos \left(\boldsymbol{r}_{j} \cdot \boldsymbol{r}_{s}\right)$, $\beta=\arccos \left(\boldsymbol{r}_{i} \cdot \boldsymbol{r}_{s}\right)$ and $\gamma=\arccos \left(\boldsymbol{r}_{i} \cdot \boldsymbol{r}_{j}\right)$, from which we find

$$
\boldsymbol{r}_{j} \cdot \boldsymbol{r}_{s}=\cos \alpha, \quad \boldsymbol{r}_{i} \cdot \boldsymbol{r}_{s}=\cos \beta, \quad \boldsymbol{r}_{i} \cdot \boldsymbol{r}_{j}=\cos \gamma .
$$

From (43) and (44) we obtain

$$
\alpha-\beta=\gamma, 0<\alpha, \beta, \gamma<\pi .
$$

We expand the vector $\boldsymbol{r}_{s}$ in terms of the system of non-coplanar unit vectors

$$
\boldsymbol{r}_{\mathrm{s}}=A \boldsymbol{r}_{i}+B \boldsymbol{r}_{j}+C \boldsymbol{r}_{n} \text {, }
$$

where

$$
\boldsymbol{r}_{n}=\frac{\boldsymbol{r}_{i} \times \boldsymbol{r}_{j}}{\left|\boldsymbol{r}_{i} \times \boldsymbol{r}_{j}\right|}=\frac{\boldsymbol{r}_{i} \times \boldsymbol{r}_{j}}{\sin \gamma}, \sin \gamma>0 .
$$

Let us calculate the coefficients $A, B, C$ in (46). Multiplying both sides of equality (46) scalarly by vectors $\boldsymbol{r}_{s}, \boldsymbol{r}_{i}$ and $\boldsymbol{r}_{j}$, also taking into account their unity, we obtain the system of equations

$$
\left\{\begin{array}{l}
\boldsymbol{r}_{s}^{2}=\left(A \boldsymbol{r}_{i}+B \boldsymbol{r}_{j}+C \boldsymbol{r}_{n}\right)^{2}=A^{2}+B^{2}+C^{2}+2 A B \cos \gamma=1, \\
\boldsymbol{r}_{s} \cdot \boldsymbol{r}_{i}=A+B \cos \gamma=\cos \beta, \\
\boldsymbol{r}_{s} \cdot \boldsymbol{r}_{j}=A \cos \gamma+B=\cos \alpha
\end{array}\right.
$$

with unknowns $A, B, C$. Solving this system, we find,

$$
\begin{gathered}
A=-\frac{\sin \beta}{\sin \gamma}, \quad B=\frac{\sin \alpha}{\sin \gamma}, \\
C^{2}=1-\left(A^{2}+B^{2}+2 A B \cos \gamma\right)=1-\frac{\sin ^{2} \beta}{\sin ^{2} \gamma}-\frac{\sin ^{2} \alpha}{\sin ^{2} \gamma}+\frac{2 \sin \alpha \sin \beta \cos (\alpha-\beta)}{\sin ^{2} \gamma} .
\end{gathered}
$$

Simplifying the right-hand side of the last equality, we get $C=0$.

Thus,

$$
\boldsymbol{r}_{\mathrm{s}}=A \boldsymbol{r}_{i}+B \boldsymbol{r}_{j},
$$

that is, $\boldsymbol{r}_{i}$ is a linear combination of vectors $\boldsymbol{r}_{i}$ and $\boldsymbol{r}_{j}$ and, therefore, lies with them in the same plane.

Let us consider the case when in (39) only one factor of the right-hand side vanishes. The solution of the problem is unique and can be calculated using the Formulas (22)-(24), (30), (31), (33) and (35). In this case, in Formula (35), you need to choose a denominator that is not equal to zero.

If two factors vanish on the right-hand side of (39), then the solution to the problem is the common vector of these factors. In this case, the desired point $P_{s}$ coincides with the vertex of the $P_{1} P_{2} P_{3}$ triangle, which is the end of this vector.

The third case, when all three factors in the right part of (39) become zero, cannot be realized, since otherwise the $P_{1} P_{2} P_{3}$ triangle will be degenerate.

\section{The Problem of Lightning Discharge Coordinates and Time Finding}

One of the current actual problems is monitoring of cloud-to-ground lightning 
discharges (Hu, Zhao, \& Zhu, 2010; Koshak \& Solakievicz, 2001; Kuterin, Bulatov, \& Shlyugaev, 2014; Ramachandran, Kumar, \& Kishore, 2003; Shpadi, Inchin, Lozbin, \& Ayazbayev, 2019). To solve it, the technology of deploying a network of stations registering a low-frequency radio wave coming from lightning discharges, is often used.

If a station located at a point $P_{i}\left(\varphi_{i}, \theta_{i}\right), i=1,2, \cdots, N, N \geq 3$, on the surface of the Earth recorded at the moment $t_{i}$ a radio signal from a lightning discharge that occurred at an instant at a point $P_{s}\left(\varphi_{s}, \theta_{s}\right)$, then this event leads to the equations

$$
R_{e} \arccos \left(\boldsymbol{r}_{i} \cdot \boldsymbol{r}_{s}\right)=c\left(t_{i}-t_{s}\right),
$$

in which $\boldsymbol{r}_{i}$ and $\boldsymbol{r}_{s}$ are the unit radius vectors of the $i$-th station and the points of the lightning discharge, $R_{e}=6371.032 \mathrm{~km}$ is the average radius of the Earth, $c=299792.458 \mathrm{~km} / \mathrm{s}$ is the speed of light. Introducing the notation

$$
\alpha_{i}=\frac{c t_{i}}{R_{e}}, \Delta \alpha=-\frac{c t_{s}}{R_{e}},
$$

we obtain one of the equations of system (4).

If the number $N$ of stations in the network is more than three, then they are grouped by three stations and the calculation is performed for each group. The calculation results are summarized in order to determine the optimal location and moment of the lightning discharge, taking into account the error in measuring the time and coordinates of the stations.

The method based on the idea of recording the moment of receipt of a radio signal is called Time-of-Arrival (ToA or TOA) in the scientific literature.

Let's consider a test case illustrating calculation of parameters of a lightning discharge for a network of three stations. Table 1 shows the location of stations in Kazakhstan and the arrival time of lightning radio signals.

We assume that the lightning strike occurred at a point $P_{s}\left(57.15^{\circ}, 50.30^{\circ}\right)$ (near the city of Aktobe, Kazakhstan) at moment $t_{s}=0$. The latitude of the stations in Table 1 and the latitude of the test point $P_{s}$ are indicated in the range $\left[-90^{\circ}, 90^{\circ}\right]$, that is, in accordance with geographic norms. The latitude of these points in the calculation is converted to the range $\left[0^{\circ}, 180^{\circ}\right]$. The calculation of the fixation time $t_{i}, i=1,2,3$, of lightning radio signals was carried out using equality (48). Mathematically exact values of this calculation are rounded to the practically achievable value of $10^{-8} \mathrm{~s}$. Also, Table 1 shows the values of the parameters $\alpha_{i}$, which are calculated using Formulas (49).

Table 1. The initial data of the problem.

\begin{tabular}{ccccc}
\hline Region Stations & Longitude, deg & Latitude, deg & Arrival times, $s$ & $\alpha_{i}$ \\
\hline Taraz & 71.3980 & 42.8984 & 0.00453956 & 0.213602471 \\
Balkhash & 74.9950 & 46.8481 & 0.00455022 & 0.214104062 \\
Almaty & 76.9286 & 43.2567 & 0.00563859 & 0.265315748 \\
\hline
\end{tabular}


Using the data in Table 1 and applying Formulas (1), we obtain:

$$
\begin{gathered}
\left(\boldsymbol{r}_{1}, \boldsymbol{r}_{2}, \boldsymbol{r}_{3}\right)=\left|\begin{array}{lll}
x_{1} & y_{1} & z_{1} \\
x_{2} & y_{2} & z_{2} \\
x_{3} & y_{3} & z_{3}
\end{array}\right|=-0.004532 \neq 0, \\
\left\{\begin{array}{l}
\arccos \left(\boldsymbol{r}_{1} \cdot \boldsymbol{r}_{2}\right)-\left(\alpha_{2}-\alpha_{1}\right)=0.081523>0 \\
\arccos \left(\boldsymbol{r}_{1} \cdot \boldsymbol{r}_{3}\right)-\left(\alpha_{3}-\alpha_{1}\right)=0.019057>0, \\
\arccos \left(\boldsymbol{r}_{2} \cdot \boldsymbol{r}_{3}\right)-\left(\alpha_{3}-\alpha_{2}\right)=0.015845>0
\end{array}\right.
\end{gathered}
$$

Based on expressions (50) and (51), we conclude that conditions for solvability of problem are satisfied and the problem has two solutions.

The transformation of stations coordinates is performed by the matrix (12)

$$
\boldsymbol{Q}=\left(\begin{array}{ccc}
0.2171383 & 0.6451388 & -0.7325619 \\
-0.9477573 & 0.3189924 & 0 \\
0.2336817 & 0.6942909 & 0.6807004
\end{array}\right)
$$

The results of the transformation of coordinates are shown in Table 2. The station near the city of Taraz has been moved to the North Pole.

Further, using Formulas (22)-(24), (30), (31), (33) and (35), we successively obtain:

$$
\begin{gathered}
\left\{\begin{array}{l}
A_{\varphi}=0.005262 \\
B_{\varphi}=0.00018593, \\
C_{\varphi}=-0.0001732
\end{array}\right. \\
\Delta=-0.896286, \quad \varsigma=1.294987, \\
\left\{\begin{array}{l}
\varphi_{s}^{(1)}=-2.3060784 \\
\varphi_{s}^{(2)}=-1.3871326
\end{array}\right. \\
\left\{\begin{array}{l}
\theta_{s}^{(1)}=0.2136107 \\
\theta_{s}^{(2)}=3.0799074
\end{array}\right.
\end{gathered}
$$

Finally, after performing the inverse transformation of coordinates of desired points and transforming their latitude to the range $\left[-90^{\circ}, 90^{\circ}\right]$, we find solution to the problem. The calculation results are shown in Table 3.

Table 2. Stations location and $\alpha_{i}$ parameters.

\begin{tabular}{cccc}
\hline Region Stations & Longitude, rad & Latitude, rad & $\alpha_{i}$ \\
\hline Taraz & -2.896614 & 0 & 0.213602471 \\
Balkhash & 2.590391 & 0.082024 & 0.214104062 \\
Almaty & 1.692168 & 0.070770 & 0.265315748 \\
\hline
\end{tabular}

Table 3. Calculation results of the problem.

\begin{tabular}{ccccc}
\hline Lightning & Longitude, deg & Latitude, deg & Discharge moment, s & $\Delta \alpha$ \\
\hline 1 & 57.14928 & 50.30016 & -0.000000175 & 0.00000824035 \\
2 & -103.81341 & -43.44464 & -0.060915789 & 2.86630489513 \\
\hline
\end{tabular}


The first lightning in Table 3 corresponds to the test $P_{s}\left(57.15^{\circ}, 50.30^{\circ}\right)$, and the second point is optional. The moments of discharges were calculated using right-hand Formula (49).

All calculations are performed in the MATLAB environment.

\section{Conclusion}

1) For the geometric problem considered in the article, the necessary and sufficient conditions for it are established. Subject to their observance, this task usually has two solutions, in exceptional cases, only one.

2) The above problem can be used as a mathematical model in solving the problem of time and place of cloud-to-ground type of lightning discharge finding.

3) The values of the $\alpha_{i}$ parameters can be arbitrary, taking into account that the solution of the problem depends on their differences. It follows that the time reference point in the Time-of-Arrival method can be chosen arbitrarily, but, naturally, it should be the same for the entire network of stations.

\section{Acknowledgements}

This article was created in the frame of the scientific and technical program BR05336383 of the Ministry of Digital Development, Innovations and Aerospace Industry of the Republic of Kazakhstan.

\section{Conflicts of Interest}

The authors declare no conflicts of interest regarding the publication of this paper.

\section{References}

Beklemishev, D. V. (2008). Course of Analytical Geometry and Linear Algebra. Moscow: Fizmatlit. (In Russian)

Hu, Z. X., Zhao, W. G., \& Zhu, H. P. (2010). A Source Location Algorithm of Lightning Detection Networks in China. Annales Geophysicae, 28, 1981-1991. https://doi.org/10.5194/angeo-28-1981-2010

Kiselev, A. P. (2004). Geometry. Moscow: Fizmatlit. (In Russian)

Koshak, W. J., \& Solakiewicz, R. J. (2001). TOA Lightning Location Retrieval on Spherical and Oblate Spheroidal Earth Geometries. Journal of Atmospheric and Oceanic Technology, 18, 187-199. https://doi.org/10.1175/1520-0426(2001)018<0187:TLLROS >2.0.CO;2

Krantz, P. (2007). Spherical Trigonometry. Moscow: LKI Publishing House. (In Russian, Translation from German)

Kuterin, F. A., Bulatov, A. A., \& Shlyugaev, Yu. V. (2014). The Development of the Lightning Detection Network Based on BoltekStormTracker Hardware. XV International Conference on Atmospheric Electricity, Norman, 15-20 June 2014, 71-74.

Lozbin, A., Shpadi, Yu., \& Inchin, A. (2016). Triangles Technique for Time and Location Finding of the Lightning Discharge in Spherical Model of the Earth, Journal of Geoscience and Environment Protection, 4, 125-135. 
https://doi.org/10.4236/gep.2016.44016

Ramachandran, V., Kumar, S., \& Kishore, A. (2003). Cloud-to-Ground Lightning Location Using TOGA of Sferics. The South Pacific Journal of Natural and Applied Sciences, 21, 63-65. https://doi.org/10.1071/SP03013

Shpadi, Yu. R., Inchin, A. S., Lozbin, A. Yu., \& Ayazbayev, G. M. (2019). The Method of Differentiable Functional in a Problem of Lightning Discharge Localization and Time Moment Determination. Journal of Physics: Conference Series, 1352, 012049. https://doi.org/10.1088/1742-6596/1352/1/012049

Volynsky, B. A. (1977). Spherical Trigonometry. Moscow: Nauka. (In Russian) 